\title{
NEW DATA ON THE OCCURRENCE OF THE CRITICALLY ENDANGERED COMMON ANGELSHARK, SQUATINA SQUATINA, IN THE CROATIAN ADRIATIC SEA
}

\author{
DrašKo Holcer $^{1,2}$ \& BOJAN LAZAR ${ }^{3,4, *}$
}

${ }^{1}$ Department of Zoology, Croatian Natural History Museum, Demetrova 1, HR-10000 Zagreb, Croatia ${ }^{2}$ Blue World Institute of Marine Research and Conservation, Kaštel 24, HR-51551 Veli Lošinj, Croatia

${ }^{3}$ Department of Biodiversity, Faculty of Mathematics, Natural Sciences and Information

Technologies, University of Primorska, Glagoljaška 8, SI-6000 Koper, Slovenia

${ }^{4}$ Marine Sciences Program, Juraj Dobrila University of Pula, Zagrebačka 30, HR-52100 Pula, Croatia

*corresponding author (bojan.lazar@upr.si)

Holcer, D. \& Lazar, B.: New data on the occurrence of the critically endangered common angelshark, Squatina squatina, in the Croatian Adriatic Sea. Nat. Croat., Vol. 26, No. 2, 313-320, 2017, Zagreb.

Two out of three critically endangered species of angelsharks (genus Squatina (Dumeril, 1806)) inhabiting the Mediterranean have been recorded in the Adriatic Sea, namely smoothback angelshark $S$. oculata Bonaparte, 1840 and common angelshark S. squatina (Linnaeus, 1758). While S. oculata has been extirpated from the Adriatic Sea due to overfishing, the presence of S. squatina remained questionable and some authors propose the species is regionally extinct since the 1980s. We present new data on the occurrence of S. squatina in the Croatian Adriatic Sea based upon inspection of collections from natural history museums and literature sources as well as three new records resulting from bycatch in commercial bottom trawls in 2016 and 2017. A low overall number of records and the complete absence of the species in scientific trawl surveys conducted since 1958, indicate its low abundance and question the effectiveness of scientific surveying in detecting rare species. Our analysis showed that this formerly abundant species is still present in the Adriatic Sea, emphasizing the importance of implementing novel approaches, such as citizen-science programmes, in studying its current distribution. Although the legal framework for angelshark conservation already exists, poor implementation and lack of any species-specific conservation measures will most probably result in further population declines and extinction of S. squatina from the Adriatic Sea.

Key words: elasmobranchs, sharks, distribution, Adriatic Sea, Mediterranean Sea

Holcer, D. \& Lazar, B.: Novi podaci o pojavljivanju kritično ugroženog sklata sivca Squatina squatina u hrvatskom dijelu Jadrana. Nat. Croat., Vol. 26, No. 2, 313-320, 2017, Zagreb.

U Jadranu su zabilježene dvije od tri kritično ugrožene vrste sklatova koje žive u Sredozemnom moru (rod Squatina (Dumeril, 1806)), sklat žutan S. oculata Bonaparte, 1840 i sklat sivac S. squatina (Linnaeus, 1758). Dok je S. oculata nestala iz Jadrana zbog prevelikog izlova, prisutnost vrste S. squatina je upitna i neki autori predlažu da se vrsta proglasi regionalno izumrlom od 1980. godine. U radu donosimo nove podatke o pojavljivanju vrste $S$. squatina u hrvatskom dijelu Jadrana na temelju pregleda zbirki prirodoslovnih muzeja i literaturnih izvora te tri nova nalaza kao posljedice slučajnog ulova komercijalnim pridnenim koćaricama iz 2016 i 2017. Ukupni mali broj nalaza i potpuna odsutnost vrste u znanstvenim istraživanjima koćarenjem od 1958. ukazuju na njenu nisku brojnost i na upitni učinak znanstvenih istraživanja u pronalasku rijetkih vrsta. Naša analiza pokazuje da je ova nekad brojna vrsta još uvijek prisutna u Jadranu, uz naglasak na važnost primjene novih metoda za utvrđivanje njene trenutne rasprostranjenosti kao što su programi znanosti za građane. Iako pravni okvir za zaštitu sklata već postoji, njeno 
loše provođenje i nepostojanje bilo kakvih mjera zaštite specifičnih za tu vrstu vjerojatno će rezultirati daljnjim padom brojnosti populacije i izumiranjem sklata sivca u Jadranu.

Ključne riječi: prečnouste, morski psi, rasprostranjenost, Jadransko more, Sredozemno more

\section{INTRODUCTION}

The monophyletic angelshark genus Squatina (Chondrichthyes: Selachii: Squatinidae) comprises 22 moderately-sized (1-2 $\mathrm{m}$ in total length) benthic shark species, circumglobally distributed from temperate to tropical seas (STELBRINK et al., 2010). Although some representatives occur over a wider geographical range, the majority of species are restricted to smaller areas inhabiting continental shelfs and upper slopes to $500 \mathrm{~m}$ in depth (Compagno et al., 2005; Last \& White, 2008; Stelbrink et al., 2010). Restricted ranges are probably the result of the feeding behaviour of Squatina species, which are bottomdwelling, stationary ambush predators, although large-scale coastal movements have been reported in S. squatina (Wheeler et al., 1975) and S. californica (NAtanson \& Cailliet, 1986; Eschmeyer \& Herald, 1999; Compagno et al., 2005).

Three species of angel sharks belonging to the Eastern North Atlantic-Mediterranean-North Africa putative zoogeographical group have been recorded in the Mediterranean Sea (Compagno et al., 2005; LAST \& White, 2008): sawback angelshark, S. aculeata Cuvier, 1829, smoothback angelshark, S. oculata and common angelshark, S. squatina. All three species are classified as "Critically Endangered" (CR) on the IUCN Red List of Threatened Species, with decreasing population trends as a result of bycatch in demersal fisheries (Walker et al., 2005; Nieto et al., 2015). The distribution of S. aculeata in the Mediterranean is restricted to its western and central basins and Ionian Sea with no records from the Adriatic Sea (Soldo \& BARICHE, 2016). The presence of S. oculata and S. squatina was well documented throughout the region, including the Adriatic Sea, but both species experienced drastic declines and almost complete disappearance from many Mediterranean areas (CAPAPÉ et al., 2000; LIPEJ et al., 2004; JARDAs et al., 2008; KABASAKAL \& KABASAKAL, 2014; Ferretti et al., 2015, 2016), with S. oculata considered regionally extinct from the eastern Adriatic Sea (Croatia: JARDAS et al., 2008).

S. squatina was once reported to be abundant in the Adriatic Sea (BRUsina, 1888), sustaining a fishing fleet in the time of the Austro-Hungarian Empire. The fleet operated with specific nets ("squaenere" or "sklatare") and targeted angelsharks and other cartilaginous fish in the north of the basin. At the beginning of the twentieth century, considerable quantities of $S$. squatina were still landed in Venice and Trieste (ForTibUoni et al., 2016). However, during five basin-wide scientific trawl surveys carried out to assess benthic fish stocks between 1948 and 2005, the species was last recorded in 1958 (FERRETTI et al., 2013). Consequently, at present it is considered rare (JARDAs et al., 2008; ForTiBuONi et al., 2016), as is the case throughout the whole Mediterranean (CAPAPÉ et al., 2006; KABASAKAL \& KABASAKAL, 2014; Ferretti et al., 2015). For instance, S. squatina is considered a severely declined species in Turkey (FrickLE et al., 2007), and the species may now be absent from the waters of the Balearic Islands, where it used to be frequent (Ferretri et al., 2015). Due to local extinctions, S. squatina faced population fragmentation in most parts of its former geographic range encompassing European waters and the Mediterranean (CAVANAGH \& Gibson, 2007; IglÉsias et al., 2010; FerRetTi et al., 2015). The same pattern was documented in the Adriatic Sea, where the species is proposed to be extirpated from the northern part at least since the 1980s (ForTiBUoni et al., 2016), but is considered present in the eastern central and south Adriatic Sea, with possible disjunctions in some northern localities (JARDAs et al., 2008) (Fig. 1). However, FerRetTi et al. (2013) hypothesized its extirpation from the entire Adriatic Sea as it was not caught in scientific trawl surveys for over half a century. In this paper we present new records of $S$. squatina and discuss the status of angelsharks in the in the Adriatic Sea based upon new data and analysis of available data from literature.

\section{MATERIAL AND METHODS}

The data presented is based upon new records, literature sources and inspection of collections from natural history museums. New records were derived from fishermen, who initially reported findings of angelsharks on a Facebook group called "Croatian Trawlers." The authors contacted the fishermen directly to collect additional data on the specimens, including the sex of the individuals as well as morphometric and meristic parameters, fishing methods, landing location and photo-documentation. In addition, through search of the online catalogues, published references or contacting the collections curators data from collections in four natural history museums in Croatia (Croatian Natural History 


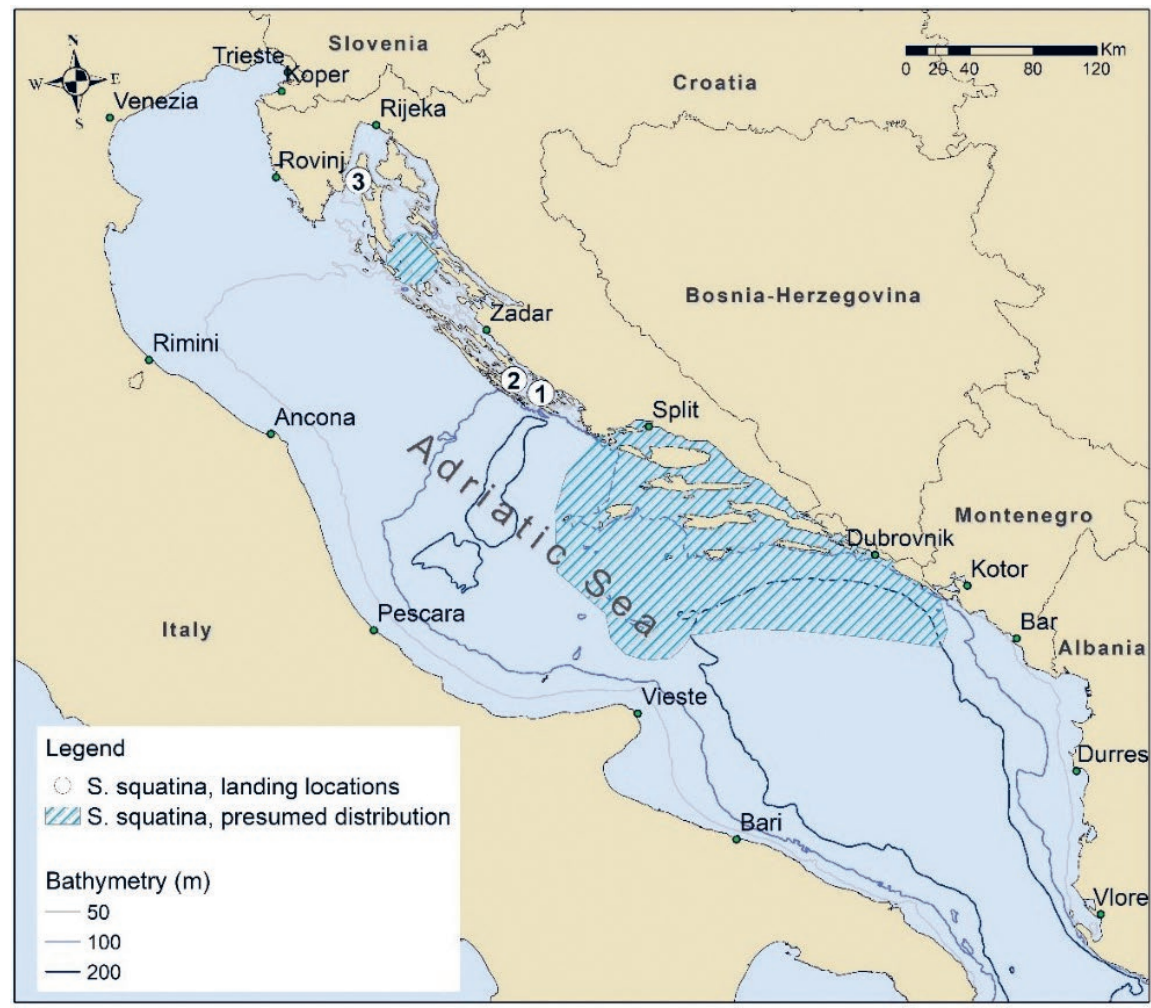

Fig. 1. Approximate landing locations (numbers correspond to Tab. 1) and presumed distribution (JARDAs et al., 2008) of common angelshark S. squatina in Croatian Adriatic Sea.

Museum (CNHM) and natural history museums of Rijeka, Split and Dubrovnik) are presented. Through online search for foreign museums holding angelshark specimens from Croatia only one was found in the Senckenberg Research Institute, Germany.

\section{RESULTS AND DISCUSSION}

We report three new findings of angelsharks from Murtersko more and Kvarner area in Croatian Adriatic Sea, all resulting from bycatch in bottom trawls in 2016 and 2017 (Tab. 1). The capture of two dead individuals was supported by photographs (Fig. 2), which enabled identification of both specimens as S. squatina (JARDAs, 1996; Lipej et al., 2004). The third record refers to an individual by-caught alive and released without any supporting documentation. Although this finding should be taken with caution, it is likely that it also refers to S. squatina due to the oral testimony of the fishermen. Data on the size of individuals are presented as reported by fishermen, while no information on the sex is available.

In addition, in museum collections we found 15 records of $S$. squatina specimens from the Croatian Adriatic Sea (Tab. 1). Fourteen of these records came from the late $19^{\text {th }}$ and early $20^{\text {th }}$ century, and only one originates from this century (2008). The majority of data accompanying the museum specimens is rather scarce and often incomplete, lacking details on time and location of finding. In addition, parts of museum material were written off from collections due to their bad state of preservation. Interestingly, in the collections of the CNHM, in addition to S. squatina specimens two other angelshark species were found. The first, S. oculata (listed as Rhina oculata; genus Rhina described by several authors is a synonym for genus Squatina (OBIS Australia, 2017)) was caught in Bakarac (North Adriatic Sea). Although S. oculata is listed as a well-known species in the Adriatic Sea (Roux, 1984; RAICEvich \& ForTI- 


\begin{tabular}{|c|c|c|c|c|c|c|c|c|c|c|c|c|c|c|c|c|c|c|c|c|c|c|}
\hline 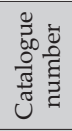 & & & & $\underset{\sim}{\stackrel{+}{+}}$ & $\overrightarrow{\vec{E}}$ & $\stackrel{\infty}{\vec{E}}$ & & & & & & & & & ৪ & 吾 & 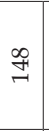 & $\stackrel{g}{\exists}$ & & & & $\stackrel{\text { है }}{:}$ \\
\hline 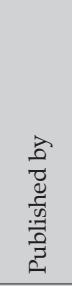 & & & & 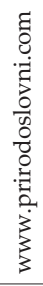 & 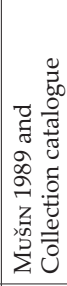 & 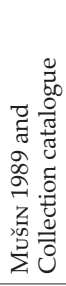 & 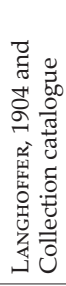 & 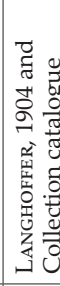 & 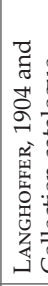 & 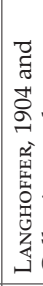 & & 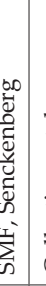 & 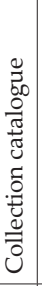 & 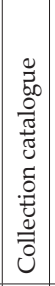 & 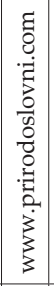 & 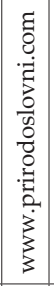 & 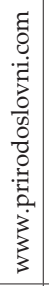 & 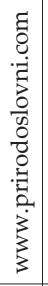 & 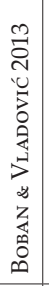 & 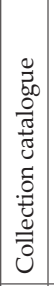 & 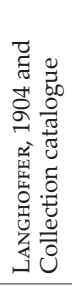 & 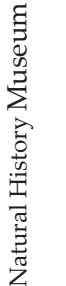 \\
\hline zั & 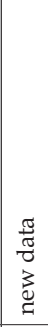 & $\begin{array}{l}\frac{\pi}{\pi} \\
\text { ] } \\
3 \\
\text { 3. }\end{array}$ & 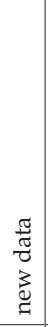 & & 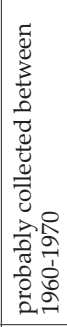 & 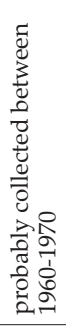 & 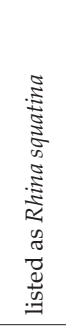 & 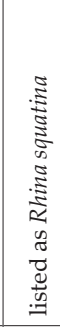 & 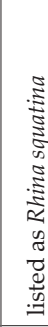 & $y$ & & & & 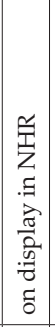 & & & & & & 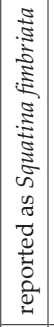 & 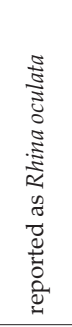 & 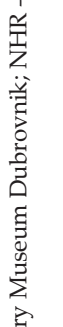 \\
\hline $\begin{array}{l}\text { 苞 } \\
\text { के }\end{array}$ & 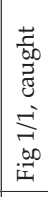 & 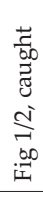 & 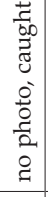 & 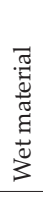 & 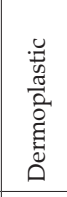 & 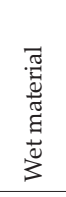 & 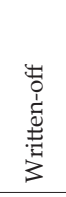 & 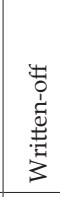 & 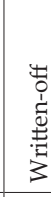 & 专 & & & $\begin{array}{l} \pm \\
0 \\
0 \\
\dot{0}\end{array}$ & 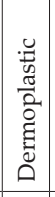 & 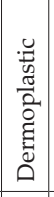 & 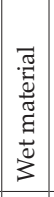 & 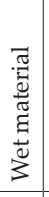 & 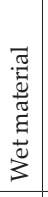 & 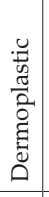 & 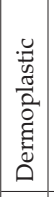 & & \\
\hline 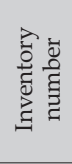 & $\begin{array}{l}\mathbb{Z} \\
\mathbb{Z} \\
\mathbb{J}\end{array}$ & $\begin{array}{l}\mathbb{Z} \\
\mathbb{J}\end{array}$ & 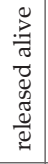 & 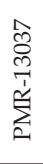 & $\stackrel{\vec{D}}{\sum_{i}}$ & $\stackrel{\infty}{\stackrel{\infty}{\infty}}$ & \&్ర & ర్రి & : & gु & & ָָ & ఫ్ర & $\begin{array}{l}\tilde{N} \\
\stackrel{N}{N}\end{array}$ & 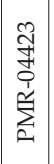 & 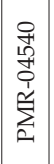 & 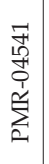 & 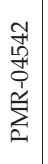 & & $\begin{array}{l}\infty \\
\text { के } \\
\infty\end{array}$ & 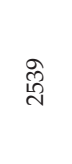 & \\
\hline 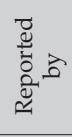 & 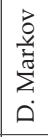 & $\begin{array}{l}\vec{\sigma} \\
\dot{z} \\
\dot{\pi} \\
\dot{\Xi}\end{array}$ & 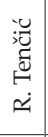 & 美 & 悬 & 見 & $\underset{\mathrm{M}}{\mathrm{M}}$ & 至 & $\underset{3}{\underline{s}}$ & $\bar{I}$ & & is & $\sum_{\text {至 }}$ & 帚 & 美 & 美 & 觉 & 寽 & 蛋 & 离 & 恋 & \\
\hline 今ँ & 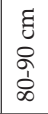 & $\begin{array}{l}\tilde{g} \\
\text { ठ }\end{array}$ & $\begin{array}{c}\tilde{\Xi} \\
\stackrel{2}{\Omega}\end{array}$ & $\begin{array}{l}\text { E } \\
\text { Ln } \\
\mathrm{n}^{-}\end{array}$ & $\begin{array}{l}\text { घ } \\
\text { 요 }\end{array}$ & $\begin{array}{l}\text { घ } \\
\text { ¿ }\end{array}$ & & & & & & & & & \begin{tabular}{l|}
$E$ \\
$E$ \\
$\swarrow$ \\
$\swarrow$
\end{tabular} & 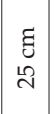 & $\begin{array}{c}\tilde{\delta} \\
\hat{~}\end{array}$ & 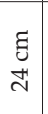 & & \begin{tabular}{l|}
$\Xi$ \\
0 \\
$\infty$
\end{tabular} & 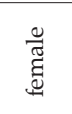 & \\
\hline 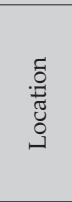 & 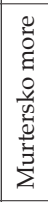 & 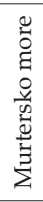 & 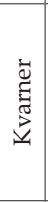 & 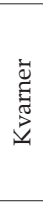 & 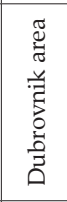 & 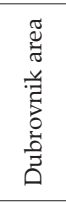 & 苛 & 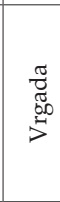 & $\begin{array}{l}\bar{\Xi} \\
\text { ఫ్ } \\
\text { ఫ్ }\end{array}$ & 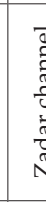 & & 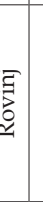 & 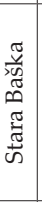 & 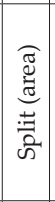 & $\begin{array}{l}\vec{E} \\
0 \\
\underline{\vec{g}} \\
\underline{\Xi}\end{array}$ & 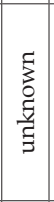 & $\begin{array}{l}\underline{\xi} \\
\vdots \\
\bar{g} \\
\vec{\Xi}\end{array}$ & 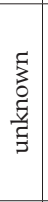 & 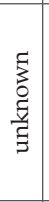 & 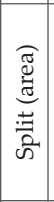 & 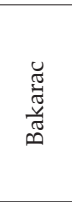 & \\
\hline صّ & 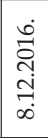 & 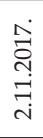 & 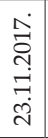 & $\begin{array}{l}\dot{0} \\
\stackrel{\overbrace{}}{0} \\
+ \\
+ \\
+\end{array}$ & $\begin{array}{l}\text { है } \\
0 \\
\vdots \\
\text { ह5 }\end{array}$ & 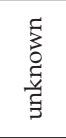 & 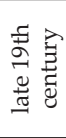 & $\underset{\sigma}{\stackrel{\sigma}{\alpha}}$ & $\begin{array}{l}\vec{\sigma} \\
\stackrel{-}{\rightrightarrows}\end{array}$ & & & ठิ่ & வூં & 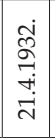 & 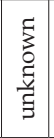 & 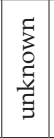 & 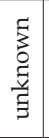 & 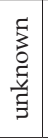 & 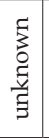 & $\mid \begin{array}{c}0 \\
\stackrel{2}{2} \\
\dot{\vec{a}} \\
\dot{\alpha}\end{array}$ & 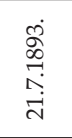 & \\
\hline $\begin{array}{l}\stackrel{\mathscr{U}}{\tilde{D}} \\
\text { के }\end{array}$ & 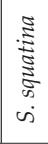 & 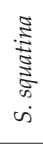 & 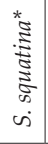 & 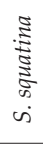 & 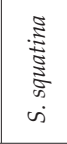 & 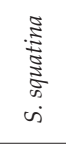 & 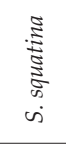 & $\frac{\mathfrak{Z}}{\mathbb{E}}$ & 吾 & 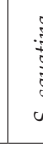 & & $\begin{array}{l}\text { 童 } \\
\text { 离 } \\
\text { क } \\
\text { is }\end{array}$ & 焉 & 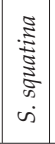 & 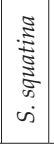 & 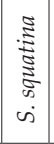 & 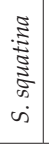 & 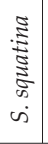 & 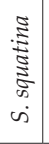 & 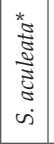 & 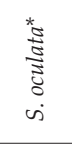 & 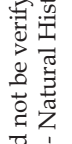 \\
\hline ż & - & N & $m$ & + & in & 0 & 1 & $\infty$ & $a$ & & & & - & $\stackrel{\oplus}{\sim}$ & $\exists$ & $\stackrel{2}{\sim}$ & $\stackrel{?}{\sim}$ & $\therefore$ & $\stackrel{\infty}{\sim}$ & 2 & 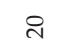 & \\
\hline
\end{tabular}


BUONI, 2013), the presence of only one record in museum collections since the $19^{\text {th }}$ century is in striking contrast to such statements. The second, S. fimbriata Müller \& Henle, 1839, what is likely a synonym for S. aculeata according to Morey et al. (2007) and CoMpagno (1984), was previously never reported from the Adriatic Sea (Notarbartolo di Sciara \& Bianchi, 1998; Soldo \& Bariche, 2016). Unfortunately, the voucher specimen of S. aculeata, although listed as present in the CNHM Ichtiology Collection (Inv. no. 3348), could not be found for species determination confirmation, so this report should be taken with some reservation.

Sharks are slow growing, late maturing and low fecundity species, hence extremely sensitive to high fishing mortality (FRISK et al., 2001; DUlvy \& FORREST, 2009). Unsustainable fishing practice currently threatens a quarter of all chondrichthian species with extinction, with Squatinidae found to be the second most threatened family of elasmobranchs in the world (Dulvy et al., 2014). Adriatic Sea species are no exceptions. The continental shelf of the Adriatic Sea is one of the hotspots for demersal fisheries in the Mediterranean, and intensive fishing has already caused a decrease in elasmobranch diversity and frequency, changing the whole elasmobranch community (Jukić-Peladić et al., 2001; Ferretti et al., 2013). After decades of commercial exploitation in the Adriatic Sea, a noticeable landing rate decline in the 1960 s caused the "economic extinction" of S. squatina (Fortibuoni et al., 2016). A severe population decline was noted throughout the range, peaking during the 1980s, when the species became completely absent from research trawl surveys and fisheries landing data. It was subsequently considered as regionally extinct (FERRETTI et al., 2013). However, reported occasional findings indicate the species is not completely extirpated from this part of its former range. In addition to the individuals reported here, there has been at least one by-caught individual in 2005 and four more were recovered in Italian waters of the northern Adriatic Sea in 2013 (ForTibuoni et al., 2016).

There is no doubt that very few reports and the complete absence of $S$. squatina from scientific trawling surveys indicate its low abundance. Obtaining data for species that faced extreme reduction in population size and are rarely caught presents a particular challenge. It also raises questions on the effectiveness of current and past methods used for scientific surveying in identifying the presence of low abundance fish species. However challenging it may be, for such species it is therefore necessary to use information from other sources, including commercial fisheries which exhibit much higher fishing effort. This particularly applies to species without commercial value. Unfortunately, two main issues stand in the way of obtaining such data. Firstly, fishermen are reluctant to report catching endangered and protected species in order to avoid any inferred or potential legal consequences. Secondly, a "shifting baseline" effect is present among younger fishermen that generally lack incentive to act upon catching angelsharks not recognising it as important (ForTibuONi et al., 2016). As there is no economic value in catching the angelsharks, whether they find it interesting enough to share their record as a "rarity" hinges on individual preference and most probably leads to many unreported catches. The discussion on the Facebook group following posting of photographs of the young fisherman's catch (Fig. 2) fully confirms such a
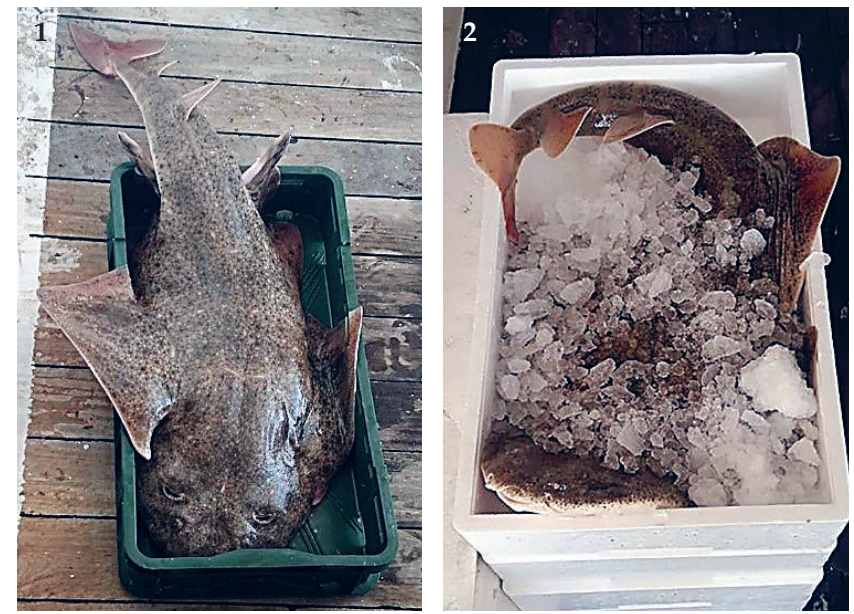

Fig. 2. Specimens of common angelshark, S. squatina, landed in Murtersko more, Eastern Adriatic Sea, Croatia on 1) 8.12.2016; 2) 2.11.2017 (numbers correspond to Tab. 1). Photo: D. Markov/FB. 
conclusion, as he was genuinely unaware of this species status and just wanted to report a "rarity" to his fellow fisherman. If researchers and managing authorities fail to obtain information on occasional catches of rare species, they are deemed to untimely declare such a species as extinct, and loose a chance to develop conservation actions that could help prevent actual extinction.

Unfortunately, although legal framework for species conservation exists, poor implementation and lack of any species-specific conservation measures will most probably result in further population decline in the Adriatic Sea. S. squatina and S. oculata are strictly protected species in Croatia (Official GAZETte, 80/13, 144/13) and their fishing is forbidden. S. aculeata is not considered present in the Adriatic Sea and is therefore not encompassed by legislation restrictions, although our report from the CNHM catalogue questions such a conclusion. All three species are listed on the List of endangered or threatened species (Annex II) of the Protocol concerning Specially Protected Areas and Biological Diversity in the Mediterranean (SPA/BD). According to Recommendation of the General Fisheries Commission for the Mediterranean (GFCM/36/2012/3), any SPA/BD Annex II-listed elasmobranch species "cannot be retained on board, transhipped, landed, transferred, stored, sold or displayed or offered for sale and must be released unharmed and alive to the extent possible" and ensured "a high protection from fishing activities". Equally, since 2009, EU Council Regulations (No. 43/2009, 23/2010, 57/2011, 44/2012, 40/2013, 43/2014, 2015/104, 2016/72, 2017/127) prohibit "retention on board, transhipping or landing of $S$. squatina for the Union fishing vessels or third-country vessels fishing in the Union waters; when accidentally caught, species shall not be harmed and shall be promptly released". Furthermore, in June 2017 the Government of Monaco sent a proposal for the inclusion of the S. squatina on Appendix I and II of the Convention on migratory species (UNEP/CMS/COP12/Doc.25.1.23).

Recently, Eastern Atlantic and Mediterranean angel shark conservation strategy has been developed aiming to, among others, increase the number of sightings reported and generate a better understanding of current distribution (Gordon et al., 2017). A novel approach to confront the issue of data gaps increasingly used for marine and coastal conservation is to use citizen science programmes (Cigliano et al., 2015). Engaging citizens and volunteers to gather information on the distribution, abundance, habitat use and population structure of elasmobranchs has been used across different species worldwide (Meyers et al., 2017). In case of S. squatina, data from coastal areas could be provided by a range of sea users (e.g. divers, snorkelers, anglers), whereas fisherman should be contacted to provide data from deeper and offshore areas. Only concerted data collection on a wider scale could shed some light on the current status of this species in the Adriatic Sea and wider Mediterranean, aiding further angelshark conservation and preventing its extinction.

\section{ACKNOWLEDGEMENTS}

The authors would like to thank fishermen Mr. D. Markov and Mr. R. Tenčić for reporting their records, museum curators Dr. S. Leiner (CNHM) and Mrs. J. Sulić Šprem (NHD) for their help in reviewing ichthyological collections, and two anonymous reviewers fot their valuable comments.

Received December 10, 2017

\section{REFERENCES}

Australian Ocean Biogeographic Information System (OBIS Australia), 2017: The Interim register of marine and nonmarine genera. https://doi.org/10.15468/6tkudz (Accessed on 17. Nov. 2017)

Boban, J. \& Vladdović, D., 2013: Rare species in Ichthyological collection of Natural History Museum Split. Natura Montenegrina 12(3-4), 599-605.

Brusina, S., 1888: Morski psi Sredozemnoga i Crljenog mora. Glasnik Hrv. narav. društva 3, 167-230.

Capapé, C., Guelorget, O., Vergne, Y., Marques, A. \& Quignard, J.P., 2006: Skates and rays (Chondrichtes) from waters off the Languedocian coast (southern France, northern Mediterranean): a historical survey and present status. Annales Ser. hist. nat. 16(2), 165-78.

CAPAPÉ, C., TomasinI, J. \& Quignard, J., 2000: Les elasmobranches pleurotrêmes de la côte du Languedoc (France méridionale): observations biologiques et démographiques. Vie et Milieu 50(2), 123-33.

Cavanagh, R.D. \& Gibson, C., 2007: Overview of the conservation status of cartilaginous fishes (Chondrichthyans) in the Mediterranean Sea. The World Conservation Union (IUCN), Gland, Switzerland, $42+$ vip. 
Cigliano, J.A., Meyer, R., Ballard, H.L., Freitag, A., Phillips, T.B. \& Wasser, A., 2015: Making marine and coastal citizen science matter. Ocean Coastal Manage. 115, 77-87.

Compagno, L., Dando, M. \& Fowler, S., 2005: A field guide to the sharks of the world. Harper Collins Publishers, London, 496p.

Compagno, L.J.V., 1984: FAO Species Catalogue. Vol. 4. Sharks of the world. An annotated and illustrated catalogue of shark species known to date. Part 1 - Hexanchiformes to Lamniformes. FAO, Rome, $249 \mathrm{p}$.

Dulvy, N.K. \& Forrest, R.E., 2009: Life histories, population dynamics and extinction risks in Chondrichthyans. In: Sharks and Their Relatives II: Biodiversity, Adaptive Physiology, and Conservation (eds. by Carrier JC, Musick JA \& Heithaus MR), pp. 635-76. CRC Press, Boca Raton, FL, USA.

Dulvy, N.K., Fowler, S.L., Musick, J.A., Cavanagh, R.D., Kyne, P.M., Harrison, L.R., Carlson, J.K., Davidson, L.N., Fordham, S.V. \& Francis, M.P., 2014: Extinction risk and conservation of the world's sharks and rays. Elife 3, e00590.

Eschmeyer, W.N. \& Herald, E.S., 1999: A field guide to Pacific coast fishes: North America. Houghton Mifflin Harcourt, Boston, USA, 342p.

Ferretti, F., Morey, G., Serena, F., Mancusi, C., Coelho, R.P., Seisay, M., Litvinov, F. \& Buscher, E., 2016: Squatina oculata (Mediterranean). In: The IUCN Red List of Threatened Species, p. e. T61418A16570000. IUCN. (Accessed on 17. Nov. 2017)

Ferretti, F., Morey, G., Serena, F., Mancusi, C., Fowler, S.L., Dipper, F. \& Ellis, J., 2015: Squatina squatina (Mediterranean). In: The IUCN Red List of Threatened Species, p. e.T39332A48933059. IUCN. (Accessed on 17. Nov. 2017)

Ferretti, F., Osio, G.C., Jenkins, C.J., Rosenberg, A.A. \& Lotze, H.K., 2013: Long-term change in a meso-predator community in response to prolonged and heterogeneous human impact. Scientific reports 3(1057), 1-11.

Fortibuoni, T., Borme, D., Franceschini, G., Giovanardi, O. \& Raicevich, S., 2016: Common, rare or extirpated? Shifting baselines for common angelshark, Squatina squatina (Elasmobranchii: Squatinidae), in the Northern Adriatic Sea (Mediterranean Sea). Hydrobiologia 772(1), 247-59.

Frickle, R., Bilecenoglu, M. \& Sari, H., 2007: Annotated checklist of fish and lamprey species (Gnathostoma and Petromyzontomorphi) of Turkey, including a Red List of threatened and declining species. Stuttg. Beitr. Nat. Kd. A Biology Ser. A (706), 1-169.

Frisk, M.G., Miller, T.J. \& Fogarty, M.J., 2001: Estimation and analysis of biological parameters in elasmobranch fishes: a comparative life history study. Can. J. Fish. Aquat. Sci. 58(5), 969-81.

Gordon, C., Hood, A., Lawson, J., Dulvy, N., Barker, J., Bartolí, À., Jiménez Alvarado, D. \& Meyers, E., 2017: Eastern Atlantic and Mediterranean angel shark conservation strategy. p. 8. The Shark Trust.

Iglésias, S.P., ToulhoAt, L. \& Sellos, D.Y., 2010: Taxonomic confusion and market mislabelling of threatened skates: important consequences for their conservation status. Aquat. Conserv.-Mar. Freshw. Ecosyst. 20(3), 319-33.

JARDAS, I., 1996: Jadranska ihtiofauna [Adriatic ichthyofauna]. Školska knjiga [In Croatian], Zagreb, 531p.

Jardas, I., Pallaoro, A., Vrgoč, N., Jukić-Peladić, S. \& Dadić, V., 2008: Crvena knjiga morskih riba Hrvatske. [Red book of sea fishes of Croatia.]. Ministarstvo kulture, Državni zavod za zaštitu prirode, Republika Hrvatska. [In Croatian.], Zagreb, 396p.

Jukić-Peladić, S., Vrgoč, N., Krstulović-Sifner, S., Piccinetti, C., Piccinetti-Manfrin, G., Marano, G. \& UnGARO, N., 2001: Long-term changes in demersal resources of the Adriatic Sea: comparison between trawl surveys carried out in 1948 and 1998. Fish Res. 53(1), 95-104.

KABASAKAL, H. \& KABASAKAL, Ö., 2014: Status of angelshark, Squatina squatina (Elasmobranchii: Squatiniformes: Squatinidae) in the sea of Marmara. Annales Ser. hist. nat. 24(1), 41-6.

LAnghoffer, A., 1904: Popis riba koje su prispjele narodnom zoološkom muzeju u Zagrebu do konca godine 1900. Glasnik Hrv. naravosl. društva 16, 148-169.

Last, P.R. \& White, W.T., 2008: Three new angel sharks (Chondrichthyes: Squatinidae) from the Indo-Australian region. Zootaxa 1734, 1-26.

Lipej, L., De Maddalena, A. \& Soldo, A., 2004: Sharks of the Adriatic sea. Annales Majora, Koper, 254 p.

Meyers, E.K.M., Tuya, F., Barker, J., Jiménez Alvarado, D., Castro-Hernández, J.J., Haroun, R. \& RöDDER, D., 2017: Population structure, distribution and habitat use of the critically endangered angelshark, Squatina squatina, in the Canary Islands. Aquat. Conserv.-Mar. Freshw. Ecosyst., 1-12. 
Morey, G., Serena, F., Mancusi, C., Coelho, R., Seisay, M., Litvinov, F. \& Dulvy, N., 2007: Squatina aculeata. In: The IUCN Red List of Threatened Species, p. e.T61417A12477164. IUCN. (Accessed on 17. Nov. 2017)

MušIN, D., 1989: Ichthyological collection (Cyclostomata, Selachii, Osteichtyes) of the Natural history museum of the Biological Institute, Dubrovnik. Zbornik Matice srpske za prirodne nauke 76, 137-168.

Natanson, L.J. \& Cailliet, G.M., 1986: Reproduction and development of the Pacific angel shark, Squatina californica, off Santa Barbara, California. Copeia 1986(4), 987-94.

Nieto, A., Ralph, G.M., Comeros-Raynal, M.T., Kemp, J., García Criado, M., Allen, D.J., Dulvy, N.K., Walls, R.H.L., Russell, B., Pollard, D., García, S., Craig, M., Collette, B.B., Pollom, R., Biscoito, M., Labbish Chao, N., Abella, A., Afonso, P., Álvarez, H. \& et Al., 2015: European Red List of marine fishes. Publications Office of the European Union, Luxembourg, 88p.

Notarbartolo di Sciara, G. \& Bianchi, I., 1998: Guida degli squali e delle razze del Mediterraneo. Franco Muzzio Editore, Padova, 388 p.

Raicevich, S. \& Fortibuoni, T., 2013: Assessing neoextirpations in the Adriatic Sea: an historical ecology approach. In: CIESM Workshop Monographs, pp. 97-111. CIESM, Monaco.

Roux, C., 1984: Squatinidae. In: Fishes of the North-East Atlantic and the Mediterranean (eds. by WHITEhead, PJP, Bauchot, ML, Hureau, JC, Nielsen, J et al.), pp. 148-52. UNESCO, Paris.

Soldo, A. \& BARICHE, M., 2016: Squatina aculeata (Mediterranean). In: The IUCN Red List of Threatened Species, p. e.T61417A16569265. IUCN.

Stelbrink, B., von Rintelen, T., Cliff, G. \& Kriwet, J., 2010: Molecular systematics and global phylogeography of angel sharks (genus Squatina). Mol. Phylogenet. Evol. 54(2), 395-404.

Walker, P., Cavanagh, R., Ducroce, M. \& Fowler, S., 2005: Regional Overviews: Northeast Atlantic (including Mediterranean and Black Sea). In: Sharks, rays and chimaeras: The status of the Chondrichthyan fishes. IUCN SSC Shark specialist group (eds. by Fowler S, Cavanagh R, Camhi M, Burgess G, Cailliet G, Fordham S, Simpfendorfer C \& Musick J), pp. 71-94. IUCN, Gland, Switzerland, Cambridge, UK.

Wheeler, A., Blacker R. \& Pirie, S., 1975: Rare and little-known fishes in British seas in 1970 and 1971. J. Fish Biol. 7(2), 183-201. 